\title{
MUCOSAL DAMAGE AND RECOVERY OF THE INTESTINE AFTER PROLONGED PRESERVATION AND TRANSPLANTATION IN DOGS ${ }^{1}$
}

\author{
Izumi Takeyoshi, ${ }^{2}$ Shimin Zhang, Minoru Nomoto, Yue Zhu, YasutaKa KokUdo, Tomomi Suzuki, \\ Nobuo hamada, Aktyoshi Nemoto, Thomas E. Starzl, and Satoru Todo
}

Pittsburgh Transplantation Institute and Department of Surgery, University of Pittsburgh, Pittsburgh, Pennsylvania, 15213

\begin{abstract}
Background. Although much is known about the mucosal damage that occurs after intestinal warm ischemia and reperfusion and its recovery, little is known about the effect of cold preservation and transplantation on the mucosa. We studied the electrophysiological, biochemical, and histological changes of the intestinal mucosa after preservation for $24 \mathrm{hr}$ and subsequent transplantation.
\end{abstract}

Methods. The small intestines from adult mongrel dogs were harvested. The intestines were orthotopically autotransplanted immediately (control group) or after preservation for $24 \mathrm{hr}$ (preservation group). Jejunal and ileal tissues were taken before harvesting, at the end of preservation, $1 \mathrm{hr}$ after reperfusion, and on postoperative days $3,7,14$, and 28 . The Ussing chamber method was used to study the electrophysiologic changes. Tissue maltase, diamine oxidase, and ornithine decarboxylase were measured. A histological analysis was also performed.

Results. Control group grafts showed no evident deterioration in electrophysiology, biochemistry, or morphology. In contrast, preservation group grafts exhibited electrophysiological and biochemical degradation, complete denudation of the villi, and crypt injury (especially in the ileum) after reperfusion. Electrophysiologic function and the mucosa biochemical marker recovered within 3 days in the jejunum and within 7-14 days in the ileum; however, histological recovery of mucosal injury required 28 days in the jejunum and more than 28 days in the ileum.

Conclusions. Our study showed that despite severe destruction of mucosal integrity by prolonged preservation and transplantation, the intestinal mucosa has an enormous regenerative capacity. Our study also showed that regeneration was more pronounced in the jejunum than in the ileum.

In the last few years, patients at our institution with intestinal failure suffering complications of total parenteral

\footnotetext{
${ }^{1}$ Aided by research grants from the Veterans Administration, and by Project Grant DK-29961 from the National Institutes of Health, Bethesda, MD.

${ }^{2}$ Address correspondence to: Izumi Takeyoshi, MD, Second Department of Surgery, Gunma University School of Medicine, 3-39-15 Showa-machi, Maebashi, Gunma 371-8511, Japan.
}

nutrition have undergone successful intestinal transplantation (1-2). Because the small intestine is notoriously sensitive to ischemia, intestinal recovery and preservation techniques are important factors in successful intestinal transplantation (3). Whereas much is known about mucosal damage and recovery after intestinal warm ischemia, little is known about the effects that cold preservation and transplantation have on the mucosa (3-6). Lillehei et al. (7) were the first to successfully preserve and transplant canine small bowel, but they were not able to prolong ischemic storage of small bowel beyond $5 \mathrm{hr}$ by simple hypothermic storage. Later, Raju et al. (8) reported successfully preserving canine small bowel for 12 and $24 \mathrm{hr}$ by using simple hypothermic storage. Neither group examined the effect of cold ischemia on the mucosa; however, Granger et al. (9) showed that intestinal reperfusion significantly exacerbates intestinal tissue injury.

Several studies have investigated the morphological recovery of the intestinal mucosa after warm and cold ischemia (10-13). A canine warm-ischemia model found that morphological mucosa recovery occurred in one day after $1 \mathrm{hr}$ of warm ischemia, and in seven days after $2 \mathrm{hr}$ of warm ischemia (10). In a 12-hr rat cold ischemia model, the morphology of the mucosa recovered 7 days after the ischemic insult (11). In a 24-hr canine cold ischemia model, Fujiwara et al. (12) were unable to observe morphological recovery of the intestinal mucosa during a 5-day follow-up after intestinal transplantation.

The purpose of our study was to evaluate the effects of prolonged ischemia and reperfusion on the intestinal mucosa and to study the process of mucosal recovery after canine intestinal autotransplantation, using electrophysiology, morphology, and biochemistry.

\section{MATERIALS AND METHODS}

Animals. Adult male and female mongrel dogs weighing $17-25 \mathrm{~kg}$ were used. The animals were given a low-residual solid meat meal and $1 \mathrm{~g}$ kanamycin sulfate daily for 5 consecutive days and then were fasted the evening before surgery. All animals received humane care in compliance with the "Principles of Laboratory Animal Care" formulated by the National Society for Medical Research and the "Guide for the Care and Use of Laboratory Animals" published by the 
National Institutes of Health (NIH publication No. 86-23, revised 1985).

Operative procedure. While under induction anesthesia with 25 $\mathrm{mg} / \mathrm{kg}$ of intravenous pentobarbital sodium, the animals were intubated and mechanically ventilated with a mixture of oxygen, nitrous oxide, and isoflurane. The arterial blood pressure and blood gasses were monitored, and electrolyte imbalances were corrected if necessary.

The abdomen was opened through a mid-line incision. At laparotomy, the entire small bowel was isolated from $5 \mathrm{~cm}$ distal to the ligament of Treitz to $5 \mathrm{~cm}$ proximal to the ileocecal valve. The isolated small bowel was removed on a vascular pedicle consisting of the superior mesenteric artery (SMA) and the superior mesenteric vein (SMV) and was transferred to a back table in ice slush. The vasculature of the graft was immediately flushed with $1 \mathrm{~L}$ of cold lactated Ringer's solution containing $3000 \mathrm{U} / \mathrm{L}$ of heparin. The warm ischemia time of the graft was less than $30 \mathrm{sec}$. After vascular flushing, the lumen of the graft was irrigated with $1 \mathrm{~L}$ of cold lactated Ringer's solution containing $500 \mathrm{mg}$ of kanamycin sulfate.

The intestinal graft was orthotopically transplanted into the same animal (autotransplantation) immediately after graft removal (control group), or $24 \mathrm{hr}$ later (preservation group). For 24-hr preservation, the resected graft was placed in a sterile plastic bag filled with cold lactated Ringer's solution which was sealed and stored at $4^{\circ} \mathrm{C}$ until transplantation. Preservation group animals received an endto-end jejunoileostomy after enteric resection, and were returned to individual cages and given adequate fluid and electrolyte replacement. The next day, they were reexplored for grafting.

Methods of intestinal transplantation in dogs have been described previously (13). In brief, vascular reconstruction was performed by end-to-end anastomoses of the graft SMA to the recipient SMA and of the graft SMV to the recipient SMV. Intestinal continuity was restored by end-to-end anastomoses of the graft jejunum to the recipient jejunum and the graft ileum to the recipient ileum.

Postoperative management. One gram of intravenous cephalosporin was given to the animals on the day of the operation and this dosage was continued for 5 days after transplantation. Lactated Ringer's solution (1-3 L) was given daily for 5-7 days until the animals were able to drink and eat normally. The animals were allowed to eat a low-residual liquid meal for the first 3 days, a low-residual solid meat meal until postoperative day 7 , and were given standard kennel food thereafter. Body weight was monitored once per week, and the condition of the stool was carefully observed. No immunosuppressive agents were administered to the animals.

Determinations. Sixty-two intestinal autotransplantations were performed in this study ( $n=28$ in the control group and $n=34$ in the preservation group). Fourteen animals died within 9 postoperative days ( $n=4$ in the control group and $n=10$ in the preservation group). The causes of animal death were arterial thrombosis $(n=12)$, venous thrombosis $(n=1)$, and intestinal anastomotic leakage $(n=1)$. No mortality from a nonfunctioning graft was seen in any of the preservation animals.

Six animals from each experimental group were sacrificed on postoperative days $3,7,14$, and 28 to collect 10 -cm segments of the proximal jejunum and distal ileum from the intestinal grafts. In addition, these same segments were taken from groups of six randomly selected animals before procurement, before transplantation, and $1 \mathrm{hr}$ after revascularization. Mucosal damage of the jejunum and ileum and its recovery after preservation and transplantation were studied electrophysiologically, biochemically, and histopathologically. Blood samples were collected every week from the animals assigned to the 28-day survival group to determine the serum levels of total protein, albumin, cholesterol, and triglyceride.

Electrophysiologic measurements. Electrophysiologic measurements were performed by essentially the same method as previously reported $(13,14)$. Specimens consisting of the mucosal and submucosal layers were separated from the seromuscular layer by blunt resection. Two specimens each from the graft ileum and jejunum, containing no Peyer's patches, were simultaneously mounted on four 13.5-mm diameter Ussing chambers (World Precision Instrument Inc., Sarasota, FL). Each surface of the specimen was continuously perfused with recirculating, oxygenated $\left(95 \% \mathrm{O}_{2}, 5 \% \mathrm{CO}_{2}\right)$ buffer solution consisting of $120 \mathrm{mM} \mathrm{NaCl}, 4.7 \mathrm{mM} \mathrm{KCl}, 1.2 \mathrm{mM} \mathrm{MgSO}_{4}$, $2.5 \mathrm{mM} \mathrm{CaCl}_{2}, 25 \mathrm{mM} \mathrm{NaHCO}_{3}$, and $1.2 \mathrm{mM} \mathrm{KH}_{2} \mathrm{PO}_{4}$ at a pH of 7.4. The recirculating reservoirs were maintained at $37^{\circ} \mathrm{C}$. Agar bridges separately connected the mucosal and submucosal compartments to voltage-sensitive and current-passing electrodes. After a 30-min equilibration period, the potential difference (PD), short-circuit current (Isc), and resistance were measured in one set (jejunum/ileum) of Ussing chambers, and changes in these values after glucose or theophylline stimulation were measured in the other set.

Biochemical analysis. Jejunal and ileal mucosa were scrapped off with a glass slide and were immediately frozen and stored in liquid nitrogen for the following assays.

Protein concentration was determined by the method of Bradford (15) with a Bio-Rad protein assay kit (Bio-Rad, Richmond, CA), using bovine serum albumin as a protein standard. Tissue maltase activity was measured by the method of Dahlqvist (16). We determined glucose levels contained in the homogenate and those released from a mixture of the tissue homogenate and a substrate solution $(2 \%$ maltose in $0.1 \mathrm{~mol} / \mathrm{L}$ maleate buffer, $\mathrm{pH} 6.0$ ) by using glucose oxidase (type 2-S, Sigma Chemical, St. Louis, MO). The tissue concentration of diamine oxidase (DAO) was measured with a spectrophotofluorometer (Shimazu, Tokyo, Japan) at an activation wavelength of $425 \mathrm{~nm}$. One unit of DAO activity was defined as $1 \mu \mathrm{mol} / \mathrm{hr}$ at $37^{\circ} \mathrm{C}$. The ornithine decarboxylase (ODC) activity was measured by the method of Lapointe and Cohen (17), using radiolabeled L-ornithine $(1.0 \mathrm{mM} ; 0.1 \mu \mathrm{Ci})$ as a substrate.

Histopathology. Portions of both jejunal and ileal segments were fixed with $10 \%$ buffered formalin, embedded in paraffin, cut into $5-\mu \mathrm{m}$ sections, and stained with hematoxylin and eosin.

Statistics. Values are expressed as the mean plus the standard error of the mean. Statistical evaluations were made by variance analysis (ANOVA), using a statistical software package (StatView ${ }^{\mathrm{TM}}$, Abacus Concepts, Inc.). Probability values of $P<0.05$ were considered statistically significant.

\section{RESULTS}

Clinical course (Table 1). After intestinal transplantation, the control group animals resumed activity the next morning and started eating within 1-2 days. Recovery of the preservation group animals was slightly slower, but they started taking food beginning on the 3rd postoperative day. An important problem with the animals was the development of diarrhea. In the control group, watery diarrhea was seen for the first 7 days, but improved to normal stool within 2 weeks. In contrast, the preservation animals continued to have watery diarrhea for 2 weeks, and the stool was still soft in one-half of the animals 28 days posttransplantation. The animals with diarrhea required frequent but intermittent fluid administration for the first 2 weeks to correct moderate dehydration. Postoperative changes in body weight reflected the difference in the stool condition of the two groups. Although the body weights of control animals stabilized or increased after the 2nd postoperative week, the animals that received preserved grafts showed a persistent decline in body weight. The average body weight was significantly lower in the preservation group than in the control group at 28 days, although the animals in the two groups were equally active.

Blood chemistry (Table 1). The changes in the serum levels of total protein, albumin, cholesterol, and triglycerides in both groups of animals after intestinal transplantation are shown in Table 1. Compared with preoperative (normal) val- 
TABLE 1. General condition ${ }^{a}$

\begin{tabular}{|c|c|c|c|c|c|}
\hline & Normal & 3rd POD & 7th POD & 14th POD & 28th POD \\
\hline \multicolumn{6}{|l|}{ Body weight (\%) } \\
\hline Control & 100 & $93.19 \pm 1.71$ & $89.84 \pm 0.68$ & $87.52 \pm 1.26$ & $87.40 \pm 1.99$ \\
\hline Preservation & & $87.60 \pm 1.61$ & $86.23 \pm 2.64$ & $83.67 \pm 2.88$ & $77.26 \pm 3.10^{\#}$ \\
\hline Control & 0 & 2 & $1-2$ & 0 & 0 \\
\hline Preservation & & 2 & $1-2$ & 1 & $0-1$ \\
\hline \multicolumn{6}{|l|}{$\mathrm{TP}(\mathrm{g} / \mathrm{dl})$} \\
\hline \multicolumn{6}{|l|}{ Albumin (g/dl) } \\
\hline Control & $2.72 \pm 0.16$ & $2.05 \pm 0.10^{*}$ & $1.87 \pm 0.11^{*}$ & $2.15 \pm 0.25$ & $1.64 \pm 0.27 *$ \\
\hline Preservation & & $1.85 \pm 0.08^{*}$ & $1.75 \pm 0.31 *$ & $2.03 \pm 0.29 *$ & $1.48 \pm 0.26^{*}$ \\
\hline \multicolumn{6}{|c|}{ Cholesterol (mg/dl) } \\
\hline Control & $142.3 \pm 9.4$ & $136.0 \pm 17.3$ & $160.7 \pm 24.0$ & $124.8 \pm 15.9$ & $98.6 \pm 22.2$ \\
\hline Preservation & & $135.3 \pm 7.1$ & $110.8 \pm 15.0^{\#}$ & $95.3 \pm 16.4^{*}$ & $81.8 \pm 15.6^{*}$ \\
\hline
\end{tabular}

${ }^{a}$ Abbreviations: TP, total protein; TG, triglycerides. Diarrhea: solid, 0; mixed, 1; watery, 2.

* $P<0.05$ vs. normal; \# $P<0.05$ vs. control.

ues, all levels except triglycerides gradually decreased after surgery in both groups and were lowest at 28 days. Preservation group animals had consistently lower levels than control animals, although the difference was not statistically significant. (These results indicate that intestinal recipient animals were still malnourished 28 days after transplantation.)

Electrophysiology. In the control group, the PD and resistance of the jejunum and ileum did not change from baseline; however, the PD and resistance were significantly decreased at the end of preservation and $1 \mathrm{hr}$ after reperfusion in the preservation group. The PD and resistance of the ileum did not recover until the 14th postoperative day, whereas the values in the jejunum recovered 3 days after transplantation (Fig. 1). The Isc was almost the same as the PD. In the control group, the Gl-PD of the ileum and jejunum did not change, but the Gl-PD was significantly lower in the preser- vation group at the end of preservation and $1 \mathrm{hr}$ after reperfusion, although it recovered by 3 days after the operation. In the control group, the theophylline-stimulated PD (T-PD) of the ileum was significantly increased at the end of preservation and $1 \mathrm{hr}$ after preservation compared with normal; however, the T-PD of the jejunum did not increase. In the preservation group, the T-PD value was high on postoperative days (POD) 3-7 (Fig. 2).

Biochemistry. Maltase and DAO exist in villi and are markers for mucosal maturation (18-20). The levels decreased significantly at $1 \mathrm{hr}$ after reperfusion in the preservation group. In the jejunum, maltase and DAO recovered by 3 days after transplantation; however, recovery required 7-14 days in the ileum (Fig. 3). ODC is a marker for cell proliferation (21). The ODC peaked on POD 3 in the preservation group (Fig. 4).
Figure 1. Electrophysiology. Left: baseline potential difference. Right: resistance. In the control group, the PD and resistance of the jejunum and ileum did not change from baseline; however, the PD and resistance were significantly decreased at the end of preservation and $1 \mathrm{hr}$ after reperfusion in the preservation group. The PD and resistance of the ileum did not recover until the 14th postoperative day, whereas the values in the jejunum recovered 3 days after transplantation. C-I, control ileum; C-J, control jejunum; P.I, preservation ileum; P.J, preservation jejunum; \#, vs. normal, $P<0.05$; $\$$, vs. control, $P<0.05$.

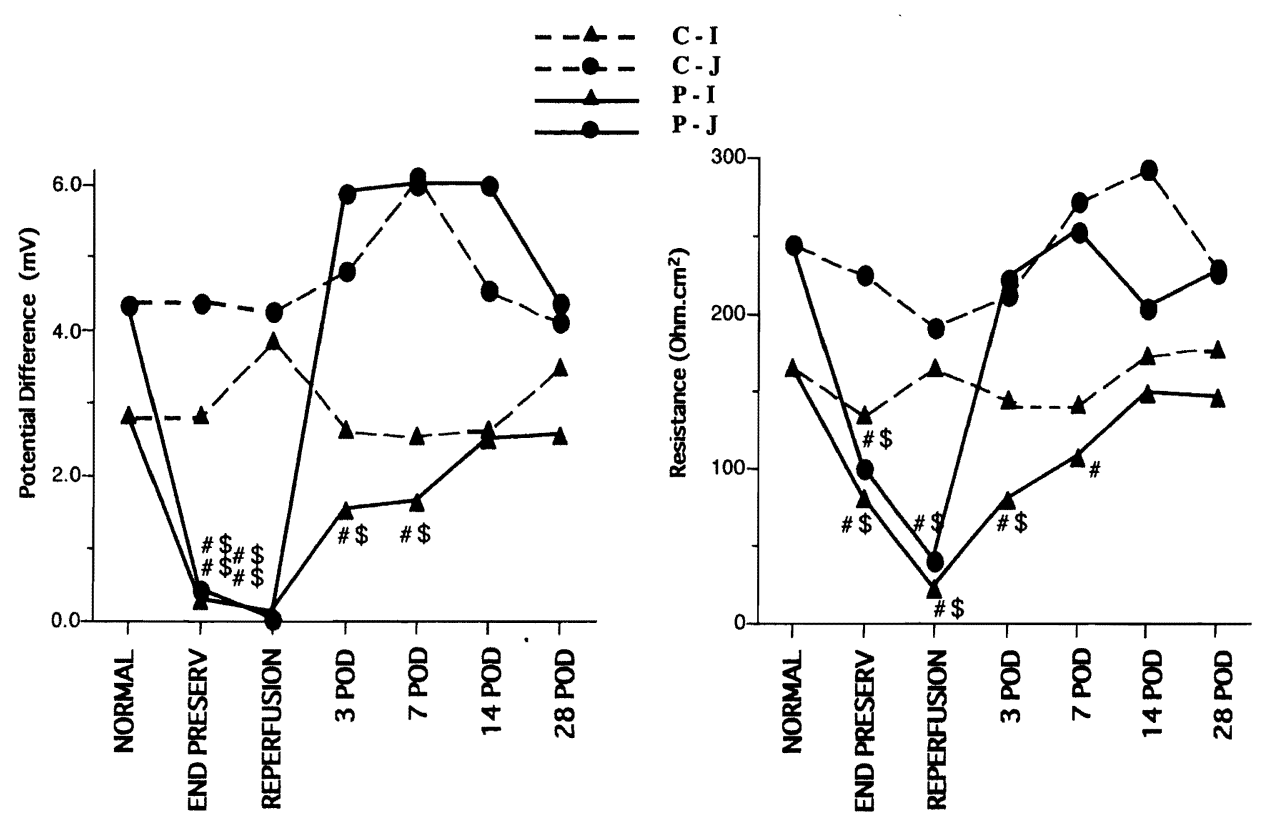




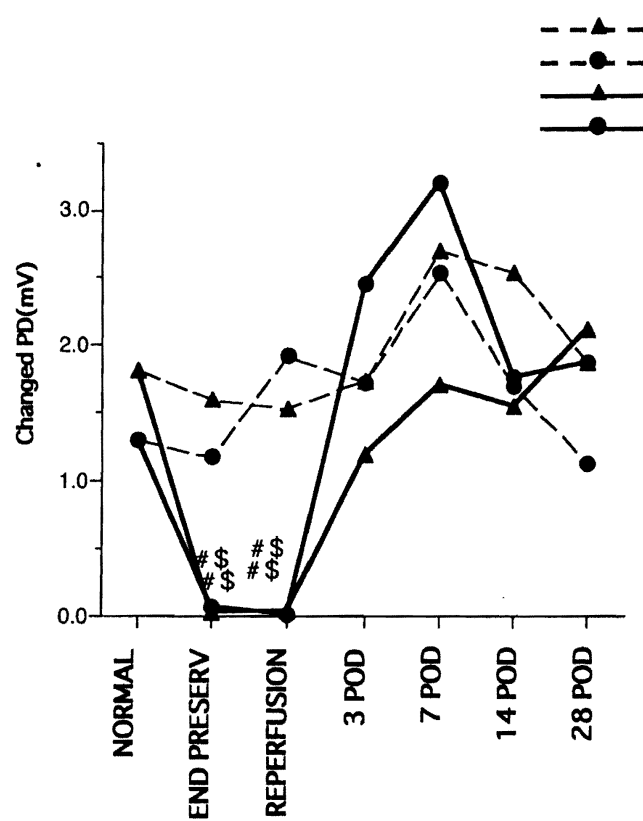

C - I

C $-\mathbf{J}$

P - I

\section{$\mathbf{P} \cdot \mathbf{J}$}

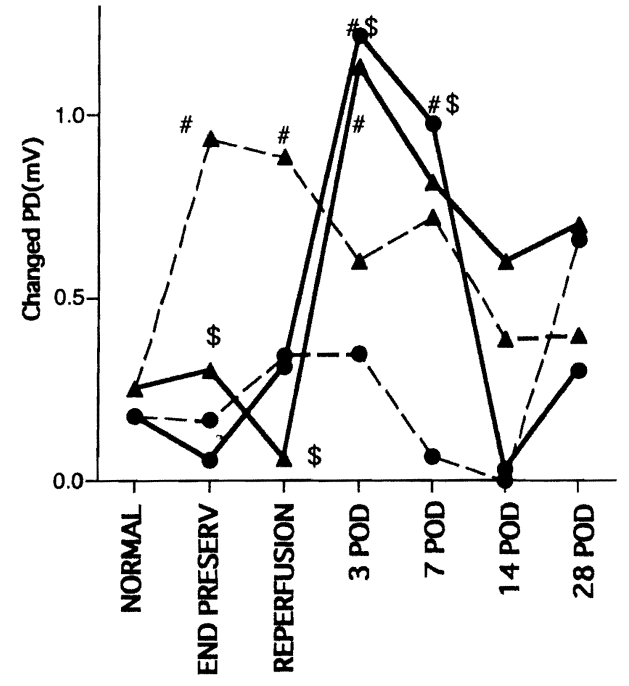

Figure 2. Electrophysiology. Left: glucose-stimulated potential difference. Right: theophylline-stimulated potential difference. In the control group, the Gl-PD of the ileum and jejunum did not change, but the Gl-PD was significantly lower in the preservation group at the end of preservation and $1 \mathrm{hr}$ after reperfusion, although it recovered by 3 days after the operation. In the control group, the theophylline-stimulated PD (T-PD) of the ileum was significantly increased at the end of preservation and $1 \mathrm{hr}$ after preservation compared with normal; however, the T-PD of the jejunum did not increase. In the preservation group, the T-PD value was high on postoperative days (POD) 3-7. C-I, control ileum; C-J, control jejunum; P-I, preservation ileum; P-J, preservation jejunum; \#, vs. normal, $P<0.05 ; \$$, vs. control, $P<0.05$

C-I

C - J

P.I
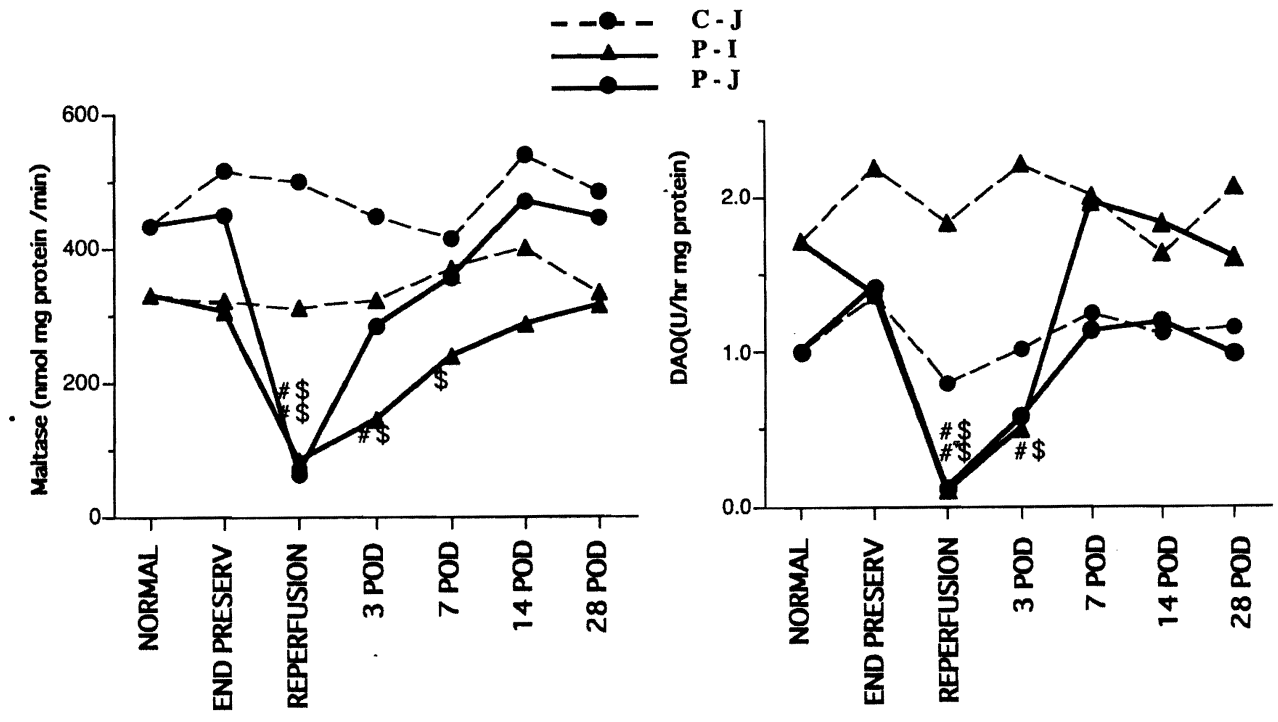

Figure 3. Biochemistry. Left: maltase activity. Right: diamine oxidase. Maltase and DAO exist levels decreased significantly at 1 $h r$ after reperfusion in the preservation group. In the jejunum, maltase and DAO recovered by 3 days after transplantation; however, recovery required 7-14 days in the ileum. C-I, control ileum; C-J, control jejunum; P-I, preservation ileum; P-J, preservation jejunum; \#, vs. normal, $P<0.05$; $\$$, vs. control, $P<0.05$.

Histopathology (Fig. 5). In the control group, the jejunum and ileum were well preserved after $1 \mathrm{hr}$ of reperfusion. The lengths of the epithelial lining cells of villi in the jejunum and ileum were almost normal at 3 days after transplantation, but the crypts were slightly atrophic and showed cystic dilation. Goblet cells and enteroendocrine cells were slightly increased in the crypt layer. At POD 7, slightly hyperplastic changes were seen in the crypt layer of the jejunum and ileum. Cystic dilation and goblet cell hyperplasia in the crypt were more predominant in the ileum than in the jejunum at POD 14. By POD 28, enteroendocrine cells had increased and appeared in the middle and upper parts of the crypt layer. This was more evident in the ileum than in the jejunum.

In the preservation group, subepithelial space or epithelial lifting was seen in both the jejunal and ileal mucosa at the end of preservation. One hour after reperfusion (Fig. 5, A and B), marked denudation and loss of villous tissue with hemorrhage were seen in the ileum predominantly, but also in the jejunum. Crypt loss was more predominant in the ileum than in the jejunum, and cell debris was seen in the crypt glands. By POD 3, regenerative epithelial cells covered the surface, but the height of the villi was reduced, and the crypts showed slight atrophy and cystic dilation. Mucosal damage was much greater in the ileum than in the jejunum. More than half the animals showed complete loss of villous tissue and marked loss of crypt glands in the ileum (Fig. 5C). On POD 7, the histological findings showed shortened, irregular villi, and healed erosions covered with regenerated epithelium were seen in some areas. On POD 14 (Fig. 5, D and E), the villous height was increasing. All of the animals had irregular villi covered with regenerative epithelium in the jejunum. The crypts showed slight atrophic and edematous changes with cystic dilation and moderate regeneration with enteroendocrine cell hyperplasia. On POD 28, the length of the villi in the jejunum was almost normal, although their shapes were irregular, whereas the ileal villi were still short. 


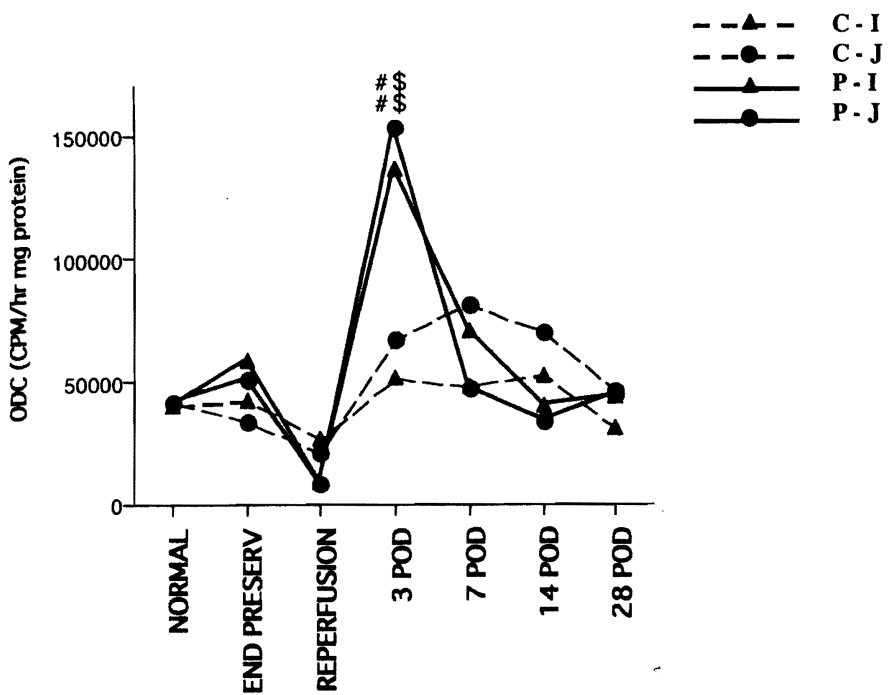

FIGURE 4. Biochemistry: ornithine decarboxylase. The ODC peaked on POD 3 in the preservation group. C-I, control ileum; C-J, control jejunum; P-I, preservation ileum; P-J, preservation jejunum; \#, vs. normal, $P<0.05$; $\$$, vs. control, $P<0.05$.

The crypts of these three animals showed slightly atrophic edematous changes, with slight regeneration and enteroeñdocrine cell hyperplasia. Two ileal tissue samples showed marked loss of villi and crypts, and the intestinal surface was covered with regenerative epithelium. The remaining crypt glands showed cystic dilation with hyperplastic changes.

\section{DISCUSSION}

During intestinal transplantation, the intestine is subjected to warm and cold ischemia. Even immediate transplantation is necessarily accompanied with short warm and cold ischemic periods. Lillehei et al. (7) were not able to extend ischemic storage of small bowel beyond $5 \mathrm{hr}$ utilizing simple hypothermia. Thirty years later, Raju et al. (8), who used simple hypothermia storage, reported successful 24-hr preservation of small bowel allotransplants. The mean survival was $22.9 \pm 27.1$ days, the 1-month survival rate was $33.3 \%$, and three dogs died within 5 postoperative days because of graft necrosis in their 24-hr storage group (8). In our preservation group, the death rate was $29.4 \%$, but no mortality from a nonfunctioning graft was seen. Although we used an autotransplantation model, our study is appropriate for examining mucosal damage and recovery of the intestine after a critical preservation time and subsequent transplantation.

In this study, we estimated the mucosal damage and recovery of the small intestine after transplantation, using general condition, survival rate, electrophysiology, biochemistry, and morphology. In electrophysiology, baseline PD or Isc is a sensitive reflection of changes in the electrical activity of mucosal cells (22-26). Resistance reflects the integrity of the tight junction between the enterocytes $(22,23)$. Gl-PD is an index of villous absorptive cells. T-PD is an index of crypt epithelial function (22). The improved outcome of the grafts was correlated with the protection of crypt cell function. T-PD (or T-Isc) was the most important indicator of graft survival $(27,28)$.
In the control group, the morphology, electrophysiology, and biochemistry of the jejunum and ileum after ischemicreperfusion injury were almost normal. Only the T-PD of the ileum was significantly increased at the end of preservation and $1 \mathrm{hr}$ after reperfusion, compared with normal. These findings suggest that the regenerative reaction of the ileal crypt has already taken effect by the end of preservation, since no other measurement detected ileal villous injury. Mucosal injury in the preservation group was not severe at the end of preservation according to the morphological and biochemical parameters, but the mucosal damage dramatically worsened after reperfusion. The histological findings after reperfusion (marked denudation, loss of villous tissue, and crypt loss) were similar to the mucosal damage seen after $2 \mathrm{hr}$ of warm ischemia-reperfusion $(3,10)$. Muller et al. (11) showed that mucosal injury worsens as ischemic time is extended and reperfusion exacerbates the preexisting damage.

Our study found that the electrophysiological and biochemical markers recovered before the morphological markers. Regenerative epithelial cells were found in the first morphological regeneration stage, and then the villous height increased after prolonged ischemia-reperfusion. This observation supports the research by Robinson et al. (10), who studied the recovery after $2 \mathrm{hr}$ of warm ischemia. Robinson et al. found a new epithelial covering capable of absorbing organic solutes in the first stages of regeneration, although the villi were considerably shorter than normal. This occurred because absorptive cells bordering the denuded areas rapidly changed shape after injury; they became flattened and sent cell projections over the denuded basement membrane. Cells from opposite sides of the denuded area came into contact, thus resealing the defect (29). Moreover, Moor et al. (30) suggested that villous contraction aided repair of the ileal epithelium after injury, and that energy-dependent, neurally mediated shortening of the villi reduces the epithelial surface area in need of resealing. In the electrophysiologic recovery, Moor et al. (30) suggested that the PD and resistance returned to near control values in parallel with growth of the epithelial covering. As cells from the sides of the original defect meet, new junctions are formed, and an epithelial barrier to passive solute and ion flow is reestablished. During the maturation process, a secretory cell is said to be gradually transformed into an absorptive element through the development of its enzymatic machinery (3). This mechanism may also induce the early recovery in electrophysiologic function. In biochemical recovery, mucosal enzymes are good markers of mucosal integrity. Because DAO activity is associated with mature villous-absorptive cells rather than proliferating crypt cells (19), DAO recovers after resealing of the enterocytes. The peak ODC activity in the preservation group was observed on POD 3, indicating the initiation of rapid cell proliferation. During the period of rapid recovery in maltase and DAO activity, the mucosal ODC activity was about 4 times normal. Because morphological recovery was not complete at the time of biochemical and electrophysiological recovery during the experimental period, the animals continued to have diarrhea, were malnourished, and lost weight.

Our study showed that the recovery of the ileum was slower than the recovery of the jejunum. This may be caused by differences in the mucosal damage. There was greater 


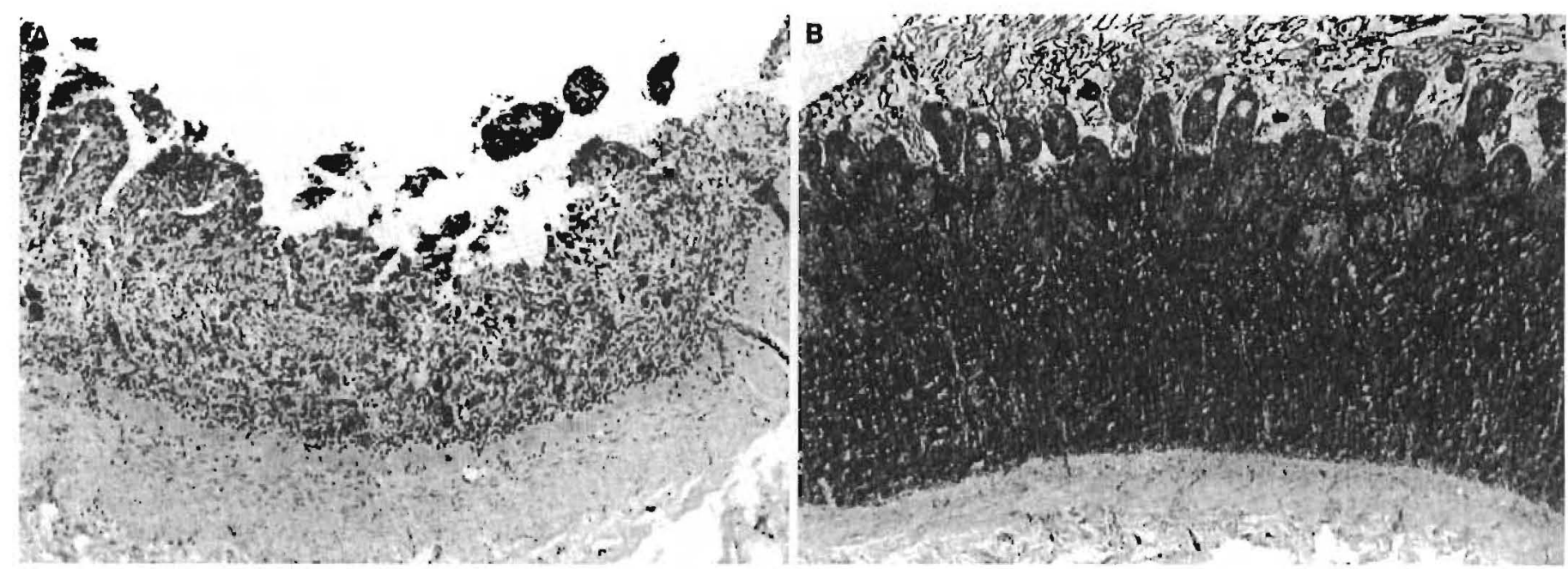

C

D
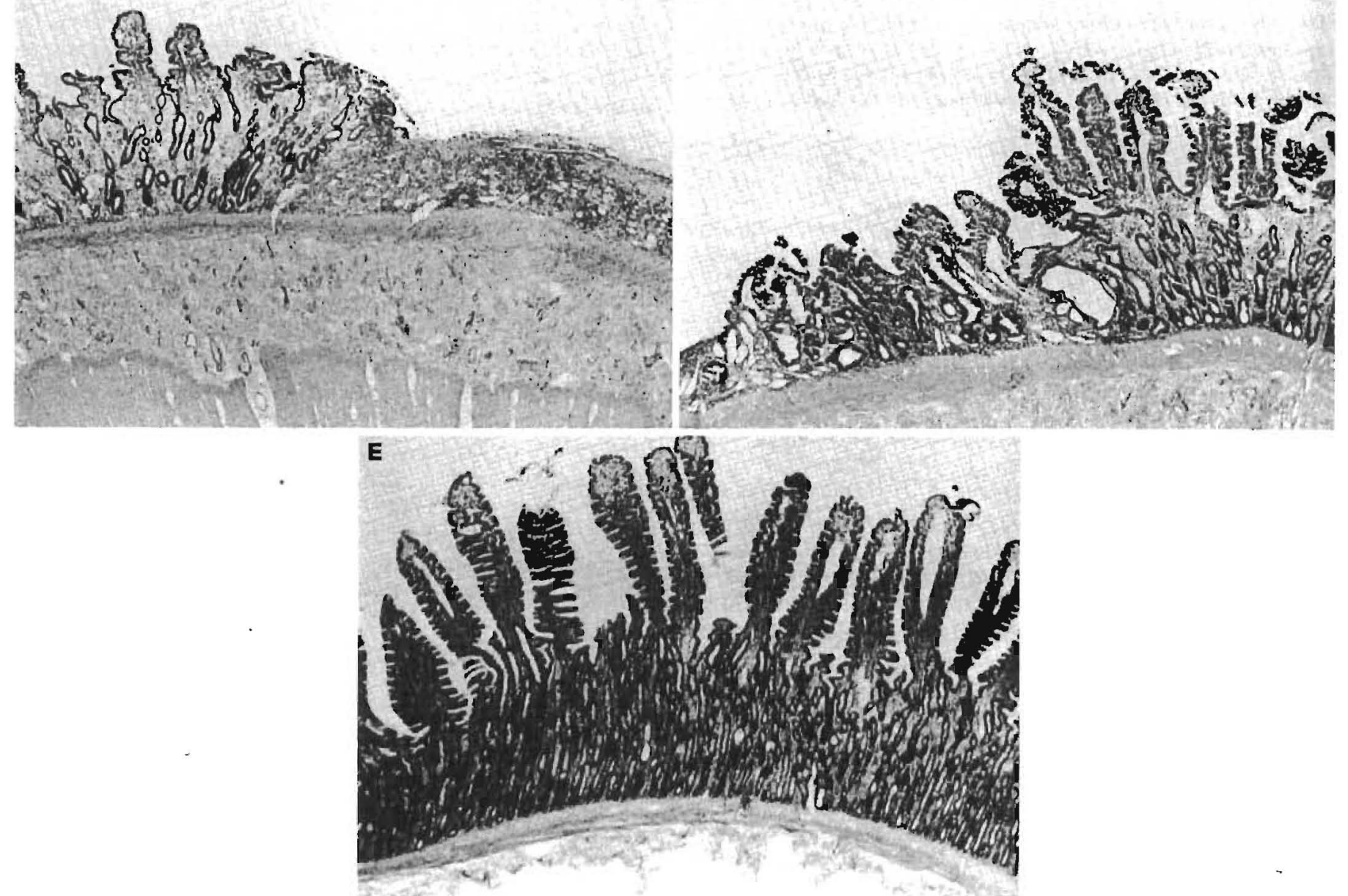

Figure 5. Histology (preservation group). A, 1 hr after reperfusion, ileum; B, 1 hr after reperfusion, jejunum; C, POD 3 ileum; D, POD 14 ileum; E, POD 14 jejunum.

crypt injury in the ileum than in the jejunum $1 \mathrm{hr}$ after reperfusion. However, it is unclear why the damage is more severe in the ileum. Whereas the damage seen after $24 \mathrm{hr}$ of cold preservation and transplantation is similar to the damage seen after $2 \mathrm{hr}$ of warm ischemia, mucosal recovery after transplantation is slower than recovery after warm ischemia. This may be caused by operative invasion and subsequent malnourished state.
We used lactated Ringer's solution as a preservation solution in this study. In another experiment, we compared the efficacy of University of Wisconsin, Euro-Collins, and lactated Ringer's solutions in preserving canine small bowel. The degree of the changes varied among the different groups, with the worst changes occurring in the lactated Ringer's solution group, moderate changes in the Euro-Collins solution group, and minimal changes in the University of Wis- 
consin solution group. Three days after transplantation, some of the Euro-Collins grafts and most of the University of Wisconsin grafts showed mucosal re-epithelialization with near-normal villous height (31).

In summary, the extensive damage to the mucosa of the canine intestine after $24 \mathrm{hr}$ of cold preservation and transplantation recovers functionally within 3-7 days in the jejunum and 7-14 days in the ileum, whereas morphological recovery takes 28 days in the jejunum and more than 28 days in the ileum. Although prolonged preservation and reperfusion causes extensive damage to the intestinal mucosa, this study shows that the intestinal mucosa has an enormous regenerative capacity, which is more pronounced in the jejunum than in the ileum.

\section{REFERENCES}

1. Todo S, Reyes J, Furukawa H, et al. Outcome analysis of 71 clinical intestinal transplantations. Ann Surg 1995; 222: 270.

2. Cicalese L, Abu-Elmagd K, Bueno J, Todo S, Reyes J. Intestinal transplantation in children after primary liver transplantation. Transplant Proc 1998; 30: 2680.

3. Robinson JWL, Mirkovitch V, Winstorfer B, Saegesser F. Response of the intestinal mucosa to ischemia. Gut 1981; 22: 512.

4. Wagner $R$, Gabbert $H, H o h n ~ P$. Ischemia and post-ischemic regeneration of the small intestinal mucosa. Virchows Arch B 1979; 31: 259.

5. Park PO, Haglund U. Regeneration of small bowel mucosa aftềr intestinal ischemia. Crit Care Med 1992; 20: 135.

6. Kummerlen C, Seiler N, Galluser M, et al. Polyamines and the recovery of intestinal morphology and function after ischemic damage in rats. Digestion 1994; 55: 168.

7. Lillehei RC, Gott B, Miller FA. The physiological response of the small bowel of the dog to ischemia including prolonged in vitro preservation of the bowel with successful replacement and survival. Ann Surg 1959; 150: 543.

8. Raju S, Fujiwara H, Lewin JR, Grogan JB. Twelve hour and twenty four hour preservation of small bowel allografts by simple hypothermia. Transplantation 1988; 45: 290.

9. Granger DN, Rutili G, McCord JM. Superoxide radicals in feline intestinal ischemia. Gastroenterology 1981; 81: 22.

10. Robinson JWL, Haroud M, Winstorfer B, Mirkovitch V. Recovery of function and structure of dog ileum and colon following two hours' acute ischemia. Eur J Clin Invest 1974; 4: 443.

11. Muller AR, Langrehr JM, Nalesnik M, et al. Mucosal glutaminase activity and histology as parameters of small bowel preservation injury. J Surg Res 1994; 56: 207.

12. Fujiwara H, Raju S, Grogan JB, Johnson WW. Organ preservation injury in small bowel transplantation. J Invest Surg 1990; 3: 23.

13. Takeyoshi I, Zhang S, Kokudo Y, et al. Effect of rejection on electrophysiologic function of canine intestinal grafts: correlation with histopathology and Na-K-ATPase activity. J Invest Surg 1995; 8: 223.
14. Takeyoshi I, Zhang S, Nakamura $\mathrm{K}$, et al. Effect of ischemia on the canine large bowel: a comparison with the small intestine. J Surg Res 1996; 62: 41.

15. Bradford MM. A rapid and sensitive method for the quantitation of microgram quantities of protein utilizing the principle of protein-dye binding. Anal Biochem 1976; 72: 248.

16. Dahlqvist A. Assay of intestinal disaccharidases. Anal Biochem 1968; 22: 99.

17. Lapointe DS, Cohen RJ. A rapid and efficient microassay of ornithine decarboxylase. Anal Biochem 1980; 109: 291.

18. Luk GD, Bayless TM, Baylin SB. Diamine oxidase. J Clin Invest 1980; 66: 66.

19. Bragg LE, Thompson JS, West WW. Intestinal diamine oxidase levels reflect ischemic injury. J Surg Res 1991; 50: 228.

20. Wollin A, Navert H, Bounous G. Effect of intestinal ischemia on diamine oxidase activity in rat intestinal tissue and blood. Gastroenterology 1981; 80: 349.

21. Luk GD, Marton LJ, Baylin SB. Ornithine decarboxylase is important in intestinal mucosal maturation and recovery from injury in rats. Science 1980; 210: 195.

22. Madara JL, Kirkman RL. Structural and functional evolution of jejunal allograft rejection in rats and ameliorating effects of cyclosporin therapy. J Clin Invest 1985; 75: 502.

23. Lee TK, Cardona MA, Kurkchubasche AG, et al. Mucosal glutamine utilization after small bowel transplantation: an electrophysiologic study. J Surg Res 1992; 52: 605.

24. Lee MD, Smith SD, Yunis EJ, Rowe MI. In vivo transmural potential difference: an early monitor of rejection in small bowel transplantation. J Pediatr Surg 1989; 24: 767.

25. Kirkman RL, Lear PA, Madara JL, Tilney NL. Small intestine transplantation in the rat: immunology and function. Surgery 1984; 96: 280

26. Meijssen MAC, Heineman E, De Bruin RWF, Ten Kate FJW, Marquet RL, Molenaar JC. Detection of canine intestinal allografts rejection by in vivo electrophysiologic monitoring. Transplantation 1991; 51: 955.

27. Kobayashi Y, Galvao FHF, Nomoto M, et al. Influence of graft perfusion pressure on graft viability after small bowel preservation and transplantation. Transplant Proc 1996; 28: 2598.

28. Kobayashi Y, Takeyoshi I. Recovery from ischemia-reperfusion injury and influence of perfusion pressure on graft viability after small bowel preservation and transplantation. Low Temp Med 1997; 23: 30.

29. Moore R, Carlson S, Madara JL. Rapid barrier restitution in an in vitro model of intestinal epithelial injury. Lab Invest 1989; 60: 237.

30. Moore R, Carlson S, Madara JL. Villus contraction aids repair of intestinal epithelium after injury. Am J Physiol 1989; 257: G274.

31. Zhang S, Takeyoshi I, Nomoto M, et al. Evaluation of solutions for canine small bowel preservation. Transplant Proc 1996; 28 : 2612.

Received 7 February 2000.

Accepted 24 March 2000. 